\title{
Case Report \\ Ovarian Torsion in a 5-Year Old: A Case Report and Review
}

\author{
Matthew F. Ryan and Bobby K. Desai \\ Department of Emergency Medicine, College of Medicine, University of Florida, P.O. Box 100186, Gainesville, FL 32610-0186, USA \\ Correspondence should be addressed to Bobby K. Desai, bdesai@ufl.edu
}

Received 5 March 2012; Accepted 22 April 2012

Academic Editors: V. Papadopoulos and M. Sand

Copyright ( 2012 M. F. Ryan and B. K. Desai. This is an open access article distributed under the Creative Commons Attribution License, which permits unrestricted use, distribution, and reproduction in any medium, provided the original work is properly cited.

\begin{abstract}
Ovarian torsion represents a true surgical emergency. Prompt diagnosis is essential to ovarian salvage, and high clinical suspicion is important in this regard. Confounding the diagnosis in general are more commonly encountered abdominal complaints in the Emergency Department (ED) such as constipation, diarrhea, and urinary tract infections and more common surgical emergencies such as appendicitis. Prompt diagnosis can be further complicated in low-risk populations such as young children. Herein, we describe the case of a 5-year-old girl with a seemingly benign presentation of abdominal pain who was diagnosed in the ED and treated for acute ovarian torsion after two prior clinic visits. A brief discussion of evaluation, treatment, and management of ovarian torsion follows.
\end{abstract}

\section{Introduction}

Ovarian torsion can be an abdominal catastrophe for women, especially if ovarian salvage is not possible. $\mathrm{Pa}$ thophysiological ramifications include ovarian loss, intraabominal infection, sepsis, and even death. The psychological impacts can also be profound [1,2]. Early diagnosis and high clinical suspicion are keys to prompt identification and definitive surgical treatment of this diagnostic dilemma. We report on a case of ovarian torsion in a five-yearold girl who initially presented to her pediatrician with nonspecific abdominal pain. There were several factors that confounded the diagnosis: firstly, was the fact that the patient had been seen and examined twice prior to presenting to the Emergency Department (ED). Secondly, the mistaken belief of the rarity of ovarian torsion within this patient population; five-year-old girls are well within the bimodal distribution for ovarian torsion. And lastly, the patient's seemingly benign presentation.

\section{Case Report}

A previously healthy five-year-old girl presented to the ED with a one-day history of fever and right lower quadrant pain. She had been evaluated by her pediatrician the previous day and was diagnosed with acute gastroenteritis after a benign physical exam and negative urinalysis. However, her symptoms progressed and her parents brought her to an after-hours urgent-care facility where a second urinalysis was performed and was again negative. The patient was again discharged with a diagnosis of gastroenteritis. The next morning the patient's pain had become more severe and she was sent to the ED by her pediatrician for further evaluation.

Upon presentation to the ED, the patient appeared ill and uncomfortable. She complained of constant pain not relieved with acetaminophen or ibuprofen. Her review of symptoms was positive for fever, one episode of vomiting, and abdominal pain. She was born full-term and has a history of asthma managed with inhaled corticosteroids and albuterol. She has two siblings who were healthy, and she had no ill contacts.

On physical exam, the patient's vital signs were within normal parameters for her age. Her oral temperature was $36.5^{\circ} \mathrm{C}$, heart rate of 90 , blood pressure of $110 / 70$, and respiratory rate of 18 , and oxygen saturation was $100 \%$ on room air. The patient's abdomen was slightly distended and tender in the right lower quadrant, pelvic, and suprapubic areas. She had some involuntary guarding but no rebound tenderness, hepatosplenomegaly, or costovertebral angle tenderness. Pain was elicited specifically at the right inguinal 


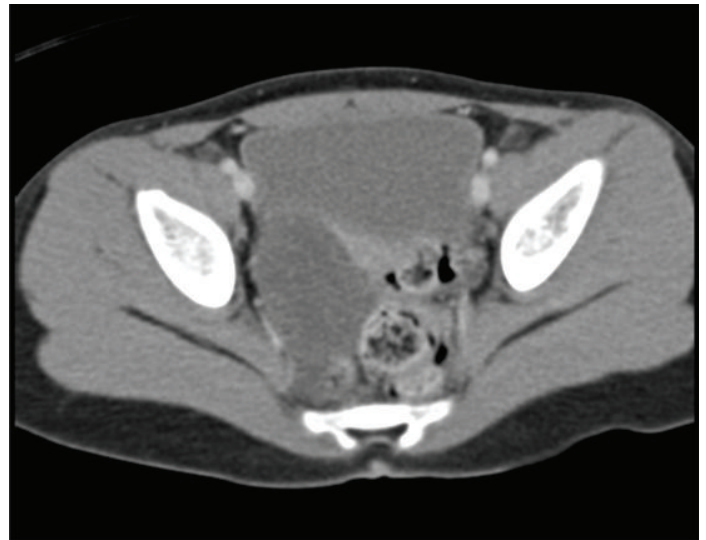

FIgURE 1: Computed tomography of the pelvis with IV contrast demonstrates a complex loculated fluid collection within right adnexa.

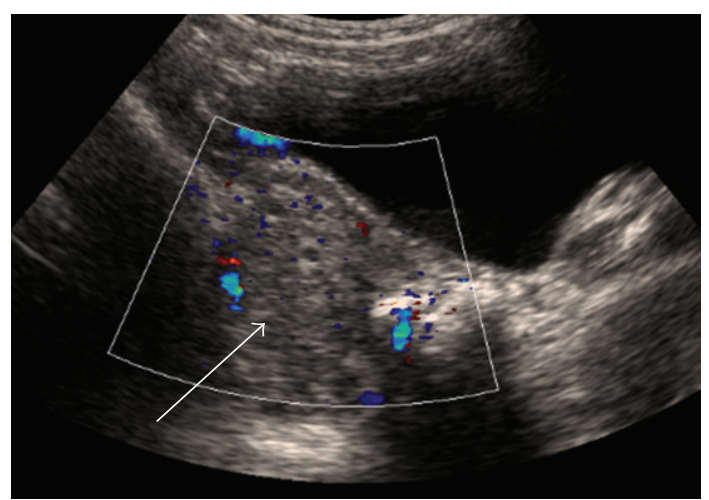

Figure 2: Doppler ultrasound of a large right ovarian cyst (estimated ovarian volume of $41 \mathrm{~cm}^{3}$ ) demonstrating arterial flow but scant venous outflow.

ligament. Classic signs for appendicitis, including Rovsing's and Psoas signs, were absent. The remainder of the exam was normal including heart tones, lung sounds, capillary refill, and skin turgor.

Her catheterized urine sample showed no protein, ketones, nitrites, or leukocyte esterase; the microscopic UA showed 30-50 red blood cells with no white cells or bacteria. The patient's white blood count was elevated at 14.6 thou $/ \mathrm{mm}^{3}$ while hemoglobin, hematocrit, and platelets were within normal levels. A basic chemistry panel was normal.

Computed tomography (CT) scan of the abdomen and pelvis with intravenous contrast demonstrated normal solid organs and normal bowel-gas pattern. A noninflammed appendix was visualized. A complex loculated fluid collection within the right adnexa and associated pelvic free fluid were seen (Figure 1). A follow-up abdominal ultrasound with Doppler, performed to further interrogate the right lower quadrant, revealed a significantly enlarged right ovary with preserved arterial flow but scant venous outflow (Figure 2). The left ovary was normal.
Exploratory laparoscopy demonstrated large right ovarian cyst $(5.5 \mathrm{~cm} \times 3.9 \mathrm{~cm} \times 3.7 \mathrm{~cm})$ with the right adnexa twisted $360^{\circ}$. The torsion was corrected and a right ovarian cyst drained. The patient was discharged on postoperative day 2 without further complications. Follow-up ultrasound performed three months later showed developing follicles in both adnexa with good arterial and venous blood flow.

\section{Discussion}

Ovarian torsion is a rare problem within the pediatric population, yet it represents a true gynecological and surgical emergency $[1,2]$. Ovarian torsion accounts for approximately $2.7 \%$ of all cases of acute abdominal pain in children $[3,4]$. Adnexal torsion is often difficult to diagnoe given the presence of nonspecific symptoms and more commonly encountered diagnoses. The presentation of adnexal torsion can mimic appendicitis, urinary tract infection, renal colic, gastroenteritis, or other conditions of acute abdominal and pelvic pain [5].

Torsion occurs more frequently in adolescents and young women [6]. For premenarchal patients, adnexal torsion occurs mostly in neonates [7]. Here, fetal and newborn ovarian cysts develop due to the high levels of circulating maternal hormones. The cysts typically resolve after birth with withdrawal of maternal hormones. In two independent sonographic studies, Orsini and coworkers [8], and Salardi et al. [9] reported the typical ovary to be solid and homogeneous in echogenicity in premenarchal girls less than six years of age. The homogeneous echogenicity is interpreted to represent ovarian parenchyma without cysts. Later, Cohen et al. [10] showed microcysts ( $<9 \mathrm{~mm}$ in greatest length) to be common in girls $2-12$ years old but macrocyts to be less common, and no cyst was seen in any girl aged 2-12 in their study of the size described herein.

Torsion occurs frequently (60\%) on the right side presumably because the sigmoid colon leaves limited space for adnexal movement $[4,11,12]$. The predominance of right-sided abdominal pain confounds diagnosis, and 38\% of children with identified adnexal masses in one study were initially diagnosed in the emergency department with appendicitis [5].

Torsion is often associated with preexisting ovarian pathology, $[6,13]$ yet large cysts (as was the case in our patient) are thought to be less likely to undergo torsion secondarily to their size and mass [14]. The large size of our patient's right ovary potentially limited the torsion, which facilitated ovarian salvage as the arterial supply was not yet compromised. Normal prepubertal ovarian volume is approximately $1-2 \mathrm{~cm}^{3}$ [15]. Our patient had an estimated right ovarian volume of $40 \mathrm{~cm}^{3}$.

The diagnosis of ovarian torsion was supported by ultrasound (approximately $87 \%$ accurate for ovarian pathology [16]). While CT may be useful in diagnosing ovarian torsion $[17,18]$, its utility here was in discerning abdominal versus gynecological pathology, for example, CT ruled out appendicitis but did not rule in, satisfactorily ovarian torsion secondary to an ovarian cyst. 


\section{Conclusion}

Prompt diagnosis and emergent surgical intervention are keys to ovary salvage, especially considering the sensitive nature of ovarian loss in the prepubescent patient. A misdiagnosis can have dire consequences including ovarian loss. Our patient was able to undergo ovarian salvage as blood flow was restored after correction. Many surgeons recommended against detorsing the ovary and prefer oophorectomy for concerns of embolization [18, 19], yet no strong evidence exists to support this claim [2, 20-22]. As demonstrated in this paper, ovarian torsion can occur at any age. Therefore a high index of suspicion coupled with radiographic evidence and clinical presentation will facilitate prompt diagnosis and ovarian salvage with significantly reduced patient comorbidity.

\section{References}

[1] L. L. Breech and P. J. A. Hillard, "Adnexal torsion in pediatric and adolescent girls," Current Opinion in Obstetrics and Gynecology, vol. 17, no. 5, pp. 483-489, 2005.

[2] D. Aziz, V. Davis, L. Allen, and J. C. Langer, "Ovarian torsion in children: is oophorectomy necessary?" Journal of Pediatric Surgery, vol. 39, no. 5, pp. 750-753, 2004.

[3] B. A. Hoey, S. P. Stawicki, W. S. Hoff, R. K. Veeramasuneni, H. Kovich, and M. D. Grossman, "Ovarian torsion associated with appendicitis in a 5-year-old girl: a case report and review of the literature," Journal of Pediatric Surgery, vol. 40, no. 9, pp. E17-E20, 2005.

[4] L. T. Hibbard, "Adnexal torsion," American Journal of Obstetrics and Gynecology, vol. 152, no. 4, pp. 456-461, 1985.

[5] A. J. Pomeranz and S. Sabnis, "Misdiagnoses of ovarian masses in children and adolescents," Pediatric Emergency Care, vol. 20, no. 3, pp. 172-174, 2004.

[6] P. A. Argenta, T. J. Yeagley, G. Ott, and S. J. Sondheimer, “Torsion of the uterine adnexa: pathologic correlations and current management trends," Journal of Reproductive Medicine, vol. 45, no. 10 , pp. 831-836, 2000.

[7] P. Bagolan, C. Giorlandino, A. Nahom et al., "The management of fetal ovarian cysts," Journal of Pediatric Surgery, vol. 37, no. 1, pp. 25-30, 2002.

[8] L. F. Orsini, S. Salardi, and G. Pilu, "Pelvic organs in premenarcheal girls: real-time ultrasonography," Radiology, vol. 153, no. 1, pp. 113-116, 1984.

[9] S. Salardi, L. F. Orsini, and E. Cacciari, "Pelvic ultrasonography in premenarcheal girls: relation to puberty and sex hormone concentrations," Archives of Disease in Childhood, vol. 60, no. 2, pp. 120-125, 1985.

[10] H. L. Cohen, P. Eisenberg, F. Mandel, and J. O. Haller, "Ovarian cysts are common in premenarchal girls: a sonographic study of 101 children 2-12 years old," American Journal of Roentgenology, vol. 159, no. 1, pp. 89-91, 1992.

[11] F. Albayram and U. M. Hamper, "Ovarian and adnexal torsion: spectrum of sonographic findings with pathologic correlation," Journal of Ultrasound in Medicine, vol. 20, no. 10, pp. 1083-1089, 2001.

[12] D. H. Nichols and D. J. Julina, "Torsion of the adnexa," Clinical Obstetrics and Gynecology, vol. 28, no. 2, pp. 375-380, 1985.

[13] A. Çelik, O. Ergün, H. Aldemir et al., "Long-term results of conservative management of adnexal torsion in children," Journal of Pediatric Surgery, vol. 40, no. 4, pp. 704-708, 2005.
[14] B. W. Warner, J. C. Kuhn, L. L. Barr, J. L. Grosfeld, R. A. Amoury, and M. W. L. Gauderer, "Conservative management of large ovarian cysts in children: the value of serial pelvic ultrasonography," Surgery, vol. 112, no. 4, pp. 749-755, 1992.

[15] L. Garel, J. Dubais, A. Grignon, D. Filiatrault, and G. Van Vliet, "US of the pediatric female pelvis: a clinical perspective," Radiographics, vol. 21, no. 6, pp. 1393-1407, 2001.

[16] S. B. Vijayaraghavan, "Sonographic whirlpool sign in ovarian torsion," Journal of Ultrasound in Medicine, vol. 23, no. 12, pp. 1643-1649, 2004.

[17] A. M. Gittleman, A. P. Price, L. Goffner, and D. S. Katz, "Ovarian torsion: CT findings in a child," Journal of Pediatric Surgery, vol. 39, no. 8, pp. 1270-1272, 2004.

[18] E. R. Kokoska, M. S. Keller, and T. R. Weber, "Acute ovarian torsion in children," American Journal of Surgery, vol. 180, no. 6, pp. 462-465, 2000.

[19] J. Mordehai, A. J. Mares, Y. Barki, R. Finaly, and I. Meizner, "Torsion of uterine adnexa in neonates and children: a report of 20 cases," Journal of Pediatric Surgery, vol. 26, no. 10, pp. 1195-1199, 1991.

[20] J. F. Anders and E. C. Powell, "Urgency of evaluation and outcome of acute ovarian torsion in pediatric patients," Archives of Pediatrics and Adolescent Medicine, vol. 159, no. 6, pp. 532535, 2005.

[21] S. E. Dolgin, M. Lublin, and E. Shlasko, "Maximizing ovarian salvage when treating idiopathic adnexal torsion," Journal of Pediatric Surgery, vol. 35, no. 4, pp. 624-626, 2000.

[22] M. Abeş and H. Sarihan, "Oophoropexy in children with ovarian torsion," European Journal of Pediatric Surgery, vol. 14, no. 3, pp. 168-171, 2004. 


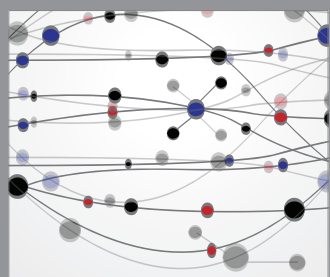

The Scientific World Journal
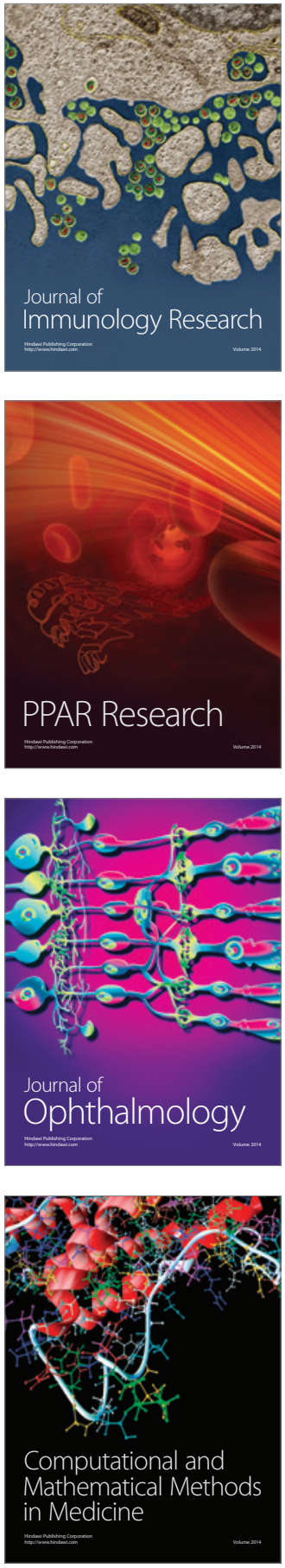

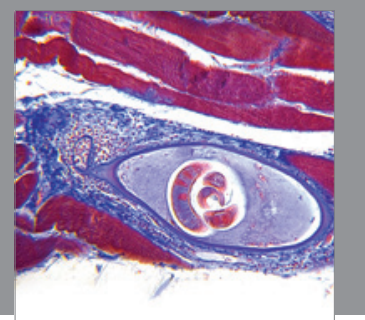

Gastroenterology

Research and Practice
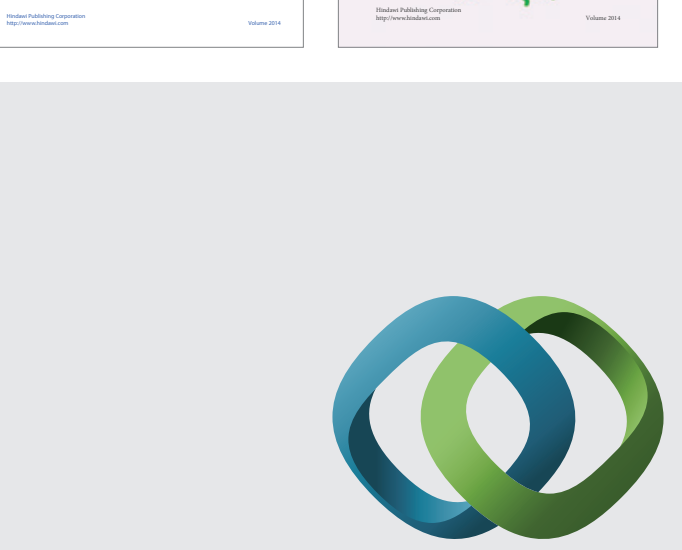

\section{Hindawi}

Submit your manuscripts at

http://www.hindawi.com
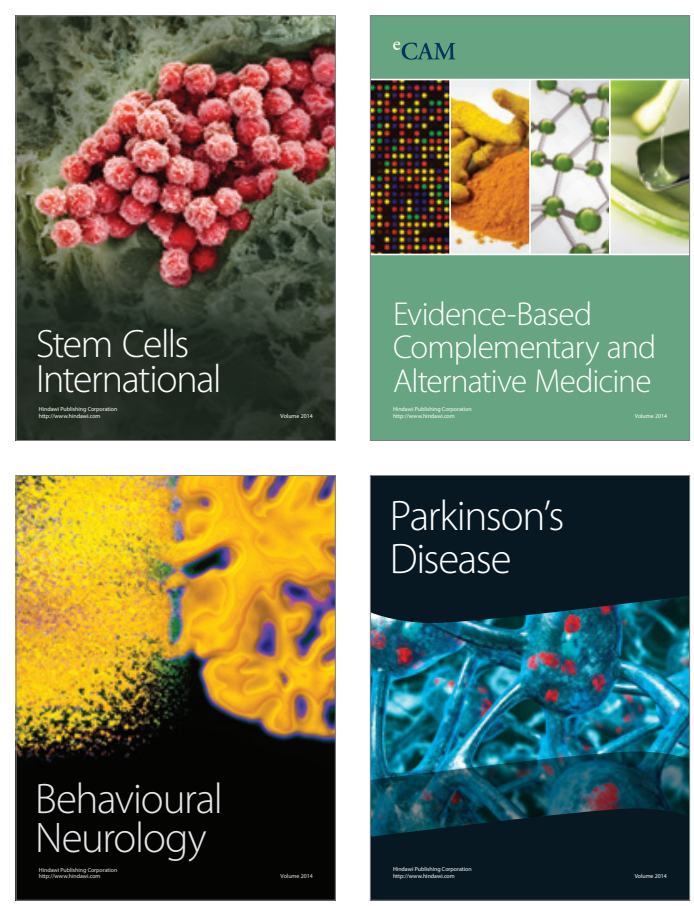

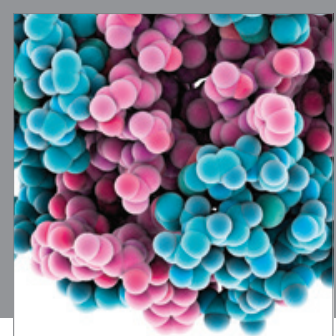

Journal of
Diabetes Research

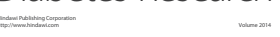

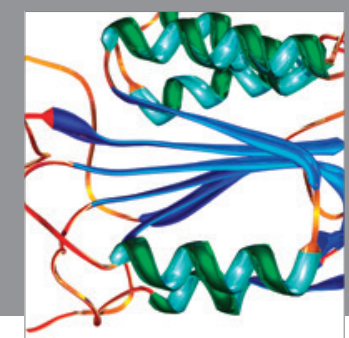

Disease Markers
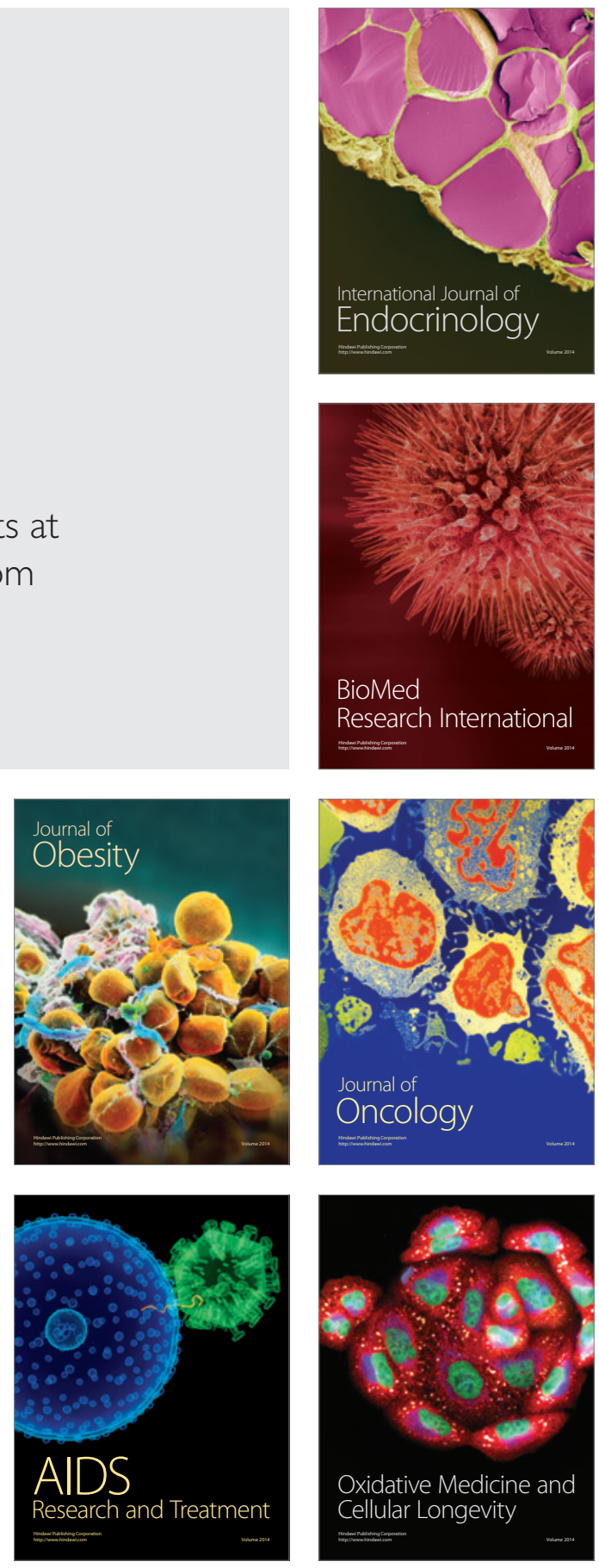